\title{
Spectral analysis of quantum Dirac systems
}

\author{
Nihal Yokus, Nimet Coskun* \\ Department of Mathematics, Karamanoglu Mehmetbey University, 70100 Karaman, Turkey.
}

Communicated by N. Shahzad

\begin{abstract}
In this study, we establish the quantum calculus analogue of the classical Dirac system. Moreover, we investigate the Jost solution, eigenvalues, spectral singularities and some quantitative properties of the spectrum of this new system. (C)2017 All rights reserved.
\end{abstract}

Keywords: Quantum equations, spectral analysis, spectral singularities. 2010 MSC: 39A70, 34L40, 47A10, 47A75.

\section{Introduction}

Spectral theory of operators is a branch of functional analysis which has a number of significant applications from quantum physics to engineering. Spectral analysis of differential operators can be traced back to Naimark [16]. Basic problems in the spectral theory of Sturm-Liouville and one dimensional Dirac type operators were discussed in the book of Levitan and Sargsjan [14]. Also, differential operators with spectral singularities have been main topic of various studies [12, 15].

Besides differential operators that we have mentioned up to here, difference operators have also become a rising research area for its possible applications to real life problems [4, 11]. Krall et al. studied some spectral properties of the non-selfadjoint discrete Schrödinger operator by taking into account the principal functions and spectral singularities [13]. Adivar and Bairamov investigated the spectrum, the spectral singularities, and the properties of the principal vectors corresponding to the spectral singularities of discrete Sturm-Liouville and discrete Dirac operators [1]. In [6], discrete Dirac operator was considered in terms of spectrum and spectral expansion.

Succeeding the developments in ordinary and discrete calculus, some new topics of quantum calculus have been introduced by Kac and Cheung [10]. This new point of view to concepts of ordinary calculus like q-derivative, q-integration etc. required the redefinition of classical equations. In particular, some problems of q-difference equations have been studied by Berg et al. [7, 8]. Adivar and Bohner introduced the quantum analogue of the classical Sturm-Liouville equation and studied the spectral properties of this

\footnotetext{
*Corresponding author

Email addresses: nyokus@kmu.edu.tr (Nihal Yokus), cannimet@kmu.edu.tr (Nimet Coskun)
} 
equation [2]. In their study, they proved that the Jost solution of this equation has analytic continuation to the lower half-plane and the finiteness of the eigenvalues and spectral singularities of the quantum difference equation is achieved as a result of this analytic continuation. Related to the study [2], principal functions of the second order quantum difference equation was treated in [3]. As a continuation of the papers [2] and [3], the presence of the spectral parameter not only in the quantum difference equation but also in the boundary condition has been considered by Aygar and Bohner [5].

The present paper was motivated by the above mentioned studies. In this paper, we let $q>1$ and use the notation

$$
q^{\mathbb{N}_{0}}:=\left\{q^{n}: n \in \mathbb{N}_{0}\right\},
$$

where $\mathbb{N}_{0}$ denotes the set of nonnegative integers. We define the Hilbert space $l_{2}\left(q^{\mathbb{N}}, \mathbb{C}^{2}\right)$ consisting of all sequences $y=\{y(t)\}=\left\{\begin{array}{l}y^{(1)}(t) \\ y^{(2)}(t)\end{array}\right\}$ with the inner product,

$$
\langle y, f\rangle_{\left(q^{\mathbb{N}}, C^{2}\right)}:=\sum_{t \in q^{\mathbb{N}}}\left(y^{(1)}(t) \overline{f^{(1)}(t)}+y^{(2)}(t) \overline{f^{(2)}(t)}\right), \quad y, f: q^{\mathbb{N}} \rightarrow \mathbb{C},
$$

and the norm

$$
\|y(t)\|_{\left(q^{\mathbb{N}}, \mathbb{C}^{2}\right)}=\left(\sum_{t \in q^{\mathbb{N}}}\left(\left|y^{(1)}(t)\right|^{2}+\left|y^{(2)}(t)\right|^{2}\right)\right)^{\frac{1}{2}} \quad \text { for } y: q^{\mathbb{N}} \rightarrow \mathbb{C} .
$$

By using the definition of quantum derivative $[2,8]$, we construct the system of equations

$$
\left\{\begin{array}{c}
\left(y^{(2)}(t)\right)^{\triangle}+p(t) y^{(1)}(t)=\frac{\lambda y^{(1)}(t)}{\mu(t)}, \\
-\left(y^{(1)}\left(\frac{t}{q}\right)\right)^{\triangle}+r(t) y^{(2)}(t)=\frac{\lambda y^{(2)}(t)}{\mu(t)},
\end{array} \quad t \in q^{\mathbb{N}},\right.
$$

which is quantum analogue of the well-known Dirac system

$$
\left(\begin{array}{cc}
0 & 1 \\
-1 & 0
\end{array}\right)\left(\begin{array}{l}
y_{1}^{\prime} \\
y_{2}^{\prime}
\end{array}\right)+\left(\begin{array}{cc}
p(x) & 0 \\
0 & q(x)
\end{array}\right)\left(\begin{array}{l}
y_{1} \\
y_{2}
\end{array}\right)=\lambda\left(\begin{array}{l}
y_{1} \\
y_{2}
\end{array}\right)
$$

introduced by Levitan and Sargsjan [14]. Therefore the system (1.1) is called a quantum Dirac system.

The purpose of this paper is investigation of the spectral properties of the non-selfadjoint boundary value problem (BVP)

$$
\left\{\begin{array}{c}
y^{(2)}(q t)-y^{(2)}(t)+p(t) \mu(t) y^{(1)}(t)=\lambda y^{(1)}(t), \\
y^{(1)}\left(\frac{t}{q}\right)-y^{(1)}(t)+r(t) \mu(t) y^{(2)}(t)=\lambda y^{(2)}(t), \quad t \in q^{\mathbb{N}}, \\
y^{(1)}(1)=0,
\end{array}\right.
$$

where $\{\mathrm{p}(\mathrm{t})\}_{\mathbf{t} \in \mathrm{q}^{\mathbb{N}}}$ and $\{\mathrm{r}(\mathrm{t})\}_{\mathbf{t} \in \mathrm{q}^{\mathbb{N}}}$ are complex sequences, $\mu(\mathrm{t})$ is graininess function and $\lambda$ is an eigenparameter. In essence, our ideas come from $[1,2]$. In the next section, we find the Jost solution and Jost function of (1.2)-(1.3). Finally, we discuss the eigenvalues and the spectral singularities of the BVP (1.2)(1.3) using the classical definitions of spectral analysis and get some quantitative properties of them under the Pavlov's condition

$$
\sup _{t \in \mathbf{q}^{\mathbb{N}}}\left\{\exp \left[\varepsilon\left(\frac{\ln t}{\ln q}\right)^{\delta}\right](|p(t) \mu(t)|+|r(t) \mu(t)|)\right\}<\infty
$$

where $\varepsilon>0, \frac{1}{2} \leqslant \delta \leqslant 1$. 


\section{Jost solution and Jost function}

Suppose that the condition

$$
\sum_{t \in q^{\mathbb{N}}} \frac{\ln t}{\ln q}(|p(t) \mu(t)|+|r(t) \mu(t)|)<\infty,
$$

is satisfied.

Theorem 2.1. Under the condition (2.1), the BVP (1.2)-(1.3) has unique solution

$$
\begin{gathered}
f^{(1)}(1, z)=e^{i \frac{z}{2}}\left[1+\sum_{r \in q^{\mathbb{N}} 0} A_{1 r}^{11} e^{i \frac{\ln r}{\ln } z}\right]-i \sum_{r \in q^{\mathbb{N}} 0} A_{1 r}^{12} e^{i \frac{\ln r}{\ln q}}, \\
f(t, z)=\left(\begin{array}{c}
f^{(1)}(t, z) \\
f^{(2)}(t, z)
\end{array}\right)=\left\{\left[\left(\begin{array}{ll}
1 & 0 \\
0 & 1
\end{array}\right)+\sum_{r \in q^{\mathbb{N}} 0} A_{t r} e^{i \frac{\ln r}{\ln q}}\right]\left(\begin{array}{c}
e^{i \frac{z}{2}} \\
-i
\end{array}\right) e^{i \ln t} z\right\},
\end{gathered}
$$

for $\lambda=2 \sin \frac{z}{2}$, where $z \in \overline{\mathbb{C}}_{+}:=\{z \in \mathbb{C}: \operatorname{Im} z \geqslant 0\}$ and

$$
A_{t r}=\left(\begin{array}{ll}
A_{t r}^{11} & A_{t r}^{12} \\
A_{t r}^{21} & A_{t r}^{22}
\end{array}\right)
$$

Proof. Substituting the function (2.3) in (1.2), we get

$$
\begin{aligned}
& A_{\mathrm{tq}}^{12}=-\sum_{s \in[\mathbf{q} t, \infty) \cap q^{\mathbb{N}}} p(s) \mu(s)+r(s) \mu(s), \\
& A_{\mathrm{tq}}^{11}=\sum_{s \in[q \mathrm{t}, \infty) \cap q^{\mathbb{N}}} p(s) \mu(s) A_{s q}^{12} \text {, } \\
& A_{\mathrm{tq}}^{22}=\sum_{s \in[\mathrm{t}, \infty) \cap \mathbf{q}^{\mathbb{N}}} p(s) \mu(s) A_{s q}^{12} \\
& A_{t q^{2}}^{12}=-r(q t) \mu(q t) A_{t q}^{11}-\sum_{s \in[q t, \infty)} \cap q^{\mathbb{N}}[p(s) \mu(s)+r(q s) \mu(q s)] A_{s q}^{11} \text {, } \\
& A_{t q}^{21}=p(t) \mu(t) A_{t q}^{11}+A_{t q}^{12}-A_{t q^{2}}^{11}
\end{aligned}
$$

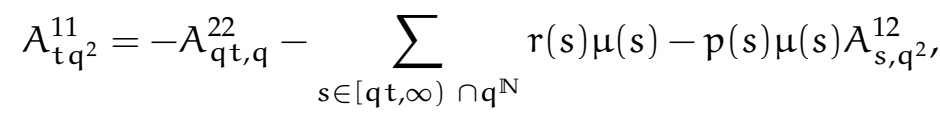

$$
\begin{aligned}
& A_{t, q^{2}}^{22}=A_{t q}^{22}-A_{t q}^{11}+A_{\frac{t}{q}, q^{2}}^{22}+r(t) \mu(t) A_{t q}^{21} \text {, }
\end{aligned}
$$

for $r \geqslant q^{3}$

$$
\begin{aligned}
& A_{t r}^{12}=A_{t r}^{21}-\sum_{s \in[t, \infty)} p(s) \mu(s) A_{s r}^{11}-r(s) \mu(s) A_{s r}^{22}, \\
& A_{t r}^{11}=-A_{t q, \frac{t}{q}}^{22}-\sum_{s \in[q t, \infty)} \sum_{q^{\mathbb{N}}} p(s) \mu(s) A_{s r}^{11}-r(s) \mu(s) A_{s, \frac{r}{q},}^{22} \\
& A_{t r}^{22}=A_{q t, \frac{t}{q}}^{22}+p(t) \mu(t) A_{t r}^{12}-A_{t, \frac{r}{q}}^{11}+A_{t r}^{11}, \\
& A_{t r}^{21}=p(t) \mu(t) A_{t r}^{11}+A_{t r}^{12}+A_{q t, \frac{t}{q}}^{21}-A_{t, q r}^{12} .
\end{aligned}
$$

Due to the condition (2.1), the series in the definition of $A_{t r}^{i j}(i, j=1,2)$ are absolutely convergent. Therefore, $A_{t r}^{i j}(i, j=1,2)$ can be uniquely determined by $p(t), r(t)$ and $\mu(t)\left(t \in q^{\mathbb{N}}\right)$, i.e., the BVP (1.2)-(1.3) for $\lambda=2 \sin \frac{z}{2}$ has the solution given by (2.2) and (2.3). 
The solution $f$ is called Jost solution of the BVP (1.2)-(1.3). Using the equalities for $A_{t r}^{i j}(i, j=1,2)$ given in Theorem 2.1, one can obtain the inequality

$$
\left|A_{\mathrm{tr}}^{i j}\right| \leqslant C \sum_{s \in\left[\mathrm{tq}^{\left[\frac{\ln r}{2 \ln q}\right]}, \infty\right) \cap q^{\mathbb{N}}}^{\infty}(|p(t) \mu(t)|+|r(t) \mu(t)|), \quad i, j=1,2,
$$

by induction, where $\left[\frac{\ln r}{2 \ln q}\right]$ is the integer part of $\frac{\ln r}{2 \ln q}, C>0$ is a constant and $t, r \in q^{\mathbb{N}}$. Therefore the function $f$ is analytic in $\mathbb{C}_{+}$, continuous in $\overline{\mathbb{C}}_{+}$and $f(z)=f(z+4 \pi)$.

\section{Eigenvalues and spectral singularities}

Let $\varphi(z)=\widetilde{\varphi}(\lambda)=\{\widetilde{\varphi}(t, \lambda)\}, t \in q^{\mathbb{N}}$ be the solution of the BVP (1.2)-(1.3) subject to the initial conditions

$$
\varphi^{(1)}(1, z)=0, \quad \varphi^{(2)}(q, z)=1 .
$$

Then $\varphi$ is entire function and

$$
\varphi(z)=\varphi(z+4 \pi)
$$

Definition 3.1. The Wronskian of two solutions $y=\{y(t, z)\}_{\mathfrak{t} \in \mathfrak{q}^{\mathbb{N}}}$ and $u=\{u(t, z)\}_{\mathfrak{t} \in q^{\mathbb{N}}}$ of the BVP (1.2)(1.3) is, for $t \in q^{\mathbb{N}}$, defined by

$$
W[y, u](t):=\{y(t, z) u(q t, z)-y(q t, z) u(t, z)\} .
$$

Let us define the semi-strips $\mathrm{P}_{0}:=\{z: z \in \mathbb{C}, z=\xi+i \tau, 0 \leqslant \xi \leqslant 4 \pi, \tau>0\}$ and $\mathrm{P}:=\mathrm{P}_{0} \cup[0,4 \pi]$. Then, from Definition 3.1 for $z \in \overline{\mathbb{C}}_{+}$, we get

$$
\begin{aligned}
W[f(z), \varphi(z)] & =\left[f^{(1)}(t, z) \varphi^{(2)}(q t, z)-f^{(2)}(q t, z) \varphi^{(1)}(t, z)\right] \\
& =f^{(1)}(1, z) .
\end{aligned}
$$

We will denote respectively the set of all eigenvalues and spectral singularities of the BVP (1.2)-(1.3) by $\sigma_{\mathrm{d}}$ and $\sigma_{\mathrm{ss}}$. It is obvious that

$$
\begin{gathered}
\sigma_{\mathrm{d}}=\left\{\lambda: \lambda=2 \sin \frac{z}{2}, z \in \mathrm{P}_{0}, \mathrm{f}^{(1)}(1, z)=0\right\}, \\
\sigma_{\mathrm{ss}}=\left\{\lambda: \lambda=2 \sin \frac{z}{2}, z \in[0,4 \pi], \mathrm{f}^{(1)}(1, z)=0\right\} \backslash\{0\} .
\end{gathered}
$$

Let

$$
F(z):=f^{(1)}(1, z) e^{-i \frac{z}{2}}
$$

then the function $F$ is analytic in $\mathbb{C}_{+}$, continuous in $\bar{C}_{+}$. Moreover, the functions $f^{(1)}$ and $F$ have the same zeros in the semi-strip P. Clearly we can write

$$
\mathrm{F}(z)=1+\sum_{\mathrm{r} \in \mathrm{q}^{\mathbb{N}_{0}}} A_{1 \mathrm{r}}^{11} e^{i \frac{\ln r}{\ln \mathrm{q}} z}-i \sum_{\mathrm{r} \in \mathrm{q}^{\mathbb{N}}{ }^{0}} A_{1 \mathrm{r}}^{12} e^{i\left(\frac{\ln \mathrm{r}}{\ln \mathrm{q}}-\frac{1}{2}\right) z},
$$

and hence,

$$
\mathrm{F}(z+4 \pi)=\mathrm{F}(z) .
$$

Using (3.1), (3.2), (3.3), we find the eigenvalues and spectral singularities of the BVP (1.2)-(1.3) as

$$
\begin{gathered}
\sigma_{\mathrm{d}}=\left\{\lambda: \lambda=2 \sin \frac{z}{2}, z \in \mathrm{P}_{0}, \mathrm{~F}(z)=0\right\}, \\
\sigma_{\mathrm{ss}}=\left\{\lambda: \lambda=2 \sin \frac{z}{2}, z \in[0,4 \pi], \mathrm{F}(z)=0\right\} \backslash\{0\} .
\end{gathered}
$$


Definition 3.2. The multiplicity of a zero of $F$ in $P$ is called the multiplicity of the corresponding eigenvalue or spectral singularity of the BVP (1.2)-(1.3).

From (3.5) and (3.6) we conclude that, in order to investigate the quantitative properties of the sets $\sigma_{\mathrm{d}}$ and $\sigma_{s s}$, we require to discuss the quantitative properties of the zeros of $F$ in $P$.

Define

$$
\begin{aligned}
& \mathrm{Q}_{1}:=\left\{z: z \in \mathrm{P}_{0}, \mathrm{~F}(z)=0\right\}, \\
& \mathrm{Q}_{2}:=\{z: z \in[0,4 \pi], \mathrm{F}(z)=0\} .
\end{aligned}
$$

We also denote the set of all limit points of $Q_{1}$ by $Q_{3}$ and the set of all zeros of $F$ with infinite multiplicity by $\mathrm{Q}_{4}$. So from (3.5), (3.6) and (3.7), we conclude the following:

$$
\begin{aligned}
\sigma_{\mathrm{d}} & =\left\{\lambda: \lambda=2 \sin \frac{z}{2}, z \in Q_{1}\right\}, \\
\sigma_{\mathrm{ss}} & =\left\{\lambda: \lambda=2 \sin \frac{z}{2}, z \in Q_{2}\right\} \backslash\{0\} .
\end{aligned}
$$

Theorem 3.3. If (2.1) holds, then

(i) The set $\mathrm{Q}_{1}$ is bounded and countable.

(ii) $\mathrm{Q}_{1} \cap \mathrm{Q}_{3}=\varnothing, \mathrm{Q}_{1} \cap \mathrm{Q}_{4}=\varnothing$.

(iii) The set $\mathrm{Q}_{2}$ is compact and $\mu\left(\mathrm{Q}_{2}\right)=0$, where $\mu$ is Lebesgue measure in the real axis.

(iv) $\mathrm{Q}_{3} \subset \mathrm{Q}_{2}, \mathrm{Q}_{4} \subset \mathrm{Q}_{2}, \mu\left(\mathrm{Q}_{3}\right)=\mu\left(\mathrm{Q}_{4}\right)=0$.

(v) $\mathrm{Q}_{3} \subset \mathrm{Q}_{4}$.

Proof. From (2.4) and (3.4), we have

$$
\mathrm{F}(z)=1+\mathrm{o}(1), \quad z \in \mathrm{P}, \quad|z| \rightarrow \infty .
$$

Equation (3.9) shows that the set $Q_{1}$ is bounded. Since $F(z)$ is analytic in $\mathbb{C}_{+}$and is a $4 \pi$ periodic function, we find that $Q_{1}$ has at most a countable number of elements. This proves (i). From the boundary uniqueness theorems of analytic functions, we obtain (ii)-(iv) [9]. Using the continuity of all derivatives of $\mathrm{F}$ on $[0,4 \pi]$, we get $(\mathrm{v})$.

From Theorem 3.3 and (3.8), we have the following.

Theorem 3.4. Under the condition (2.1),

(i) the set of eigenvalues of the BVP (1.2)-(1.3) is bounded, has at most a countable number of elements, and its limit points can lie only in $[-2,2]$.

(ii) $\sigma_{\mathrm{ss}} \subset[-2,2]$ and $\mu\left(\sigma_{\mathrm{ss}}\right)=0$.

In preparation for the next result, we assume that the complex sequences $\{p(t)\}_{t \in q^{\mathbb{N}}}$ and $\{r(t)\}_{t \in q^{\mathbb{N}}}$ satisfy

$$
\sum_{t \in q^{\mathbb{N}}} \exp \left(\varepsilon \frac{\ln t}{\ln q}\right)(|p(t) \mu(t)|+|r(t) \mu(t)|)<\infty
$$

for some $\varepsilon>0$.

Note that for $\delta=1$, the condition (1.4) reduces to (3.10).

Theorem 3.5. Under condition (3.10), the BVP (1.2)-(1.3) has a finite number of eigenvalues and spectral singularities with a finite multiplicity. 
Proof. Using (2.4) and (3.10), we obtain that

$$
|A(t, r)| \leqslant C \exp \left[-\frac{\varepsilon}{2} \frac{\ln r}{\ln q}\right], \quad t=1, \quad r \in q^{\mathbb{N}_{0}},
$$

where $C>0$ is a constant. Using (3.4) and (3.11), we observe that the function $F$ has an analytic continuation to the half-plane $\operatorname{Im} z>-\frac{\varepsilon}{2}$. Since $F$ is a $4 \pi$ periodic function, the limit points of its zeros in $P$ cannot lie in $[0,4 \pi]$. Also, we obtain that the bounded sets $Q_{1}$ and $Q_{2}$ have a finite number of elements from Theorem 3.3. Using the analyticity of $F$ in the region $\operatorname{Im} z>-\frac{\varepsilon}{2}$, we find that all zeros of $F$ in $P$ have a finite multiplicity. Therefore, we get the finiteness of the eigenvalues and the spectral singularities of the BVP (1.2)-(1.3).

Now let us suppose that

$$
\sum_{t \in q^{\mathbb{N}}} \exp \left[\varepsilon\left(\frac{\ln t}{\ln q}\right)^{\delta}\right](|p(t) \mu(t)|+|r(t) \mu(t)|)<\infty, \quad \varepsilon>0, \quad \frac{1}{2} \leqslant \delta<1,
$$

which is weaker than (3.10). It is seen that the condition (3.10) undertakes the analytic continuation of $F$ from the real axis to the lower half-plane. So the finiteness of the eigenvalues and the spectral singularities of the BVP (1.2)-(1.3) are achieved as a result of this analytic continuation. It is evident that, under the condition (3.12), the function $F$ is analytic in $\mathbb{C}_{+}$and infinitely differentiable on the real axis. However $\mathrm{F}$ does not have an analytic continuation from the real axis to the lower half-plane. Therefore, under the condition (3.12), the finiteness of the eigenvalues and the spectral singularities of the BVP (1.2)-(1.3) cannot be proved in a way similar to Theorem 3.5 .

Under the condition (3.12), to show that the eigenvalues and the spectral singularities of the BVP (1.2)-(1.3) are of finite number, we will use the following:

Theorem 3.6 ([6]). Let us assume that the $4 \pi$ periodic function $\mathrm{g}$ is analytic in $\mathbb{C}_{+}$, all of its derivatives are continuous in $\overline{\mathbb{C}}_{+}$, and

$$
\sup _{z \in \mathrm{P}}\left|g^{(k)}(z)\right| \leqslant \eta_{k}, \quad k \in \mathbb{N} \cup\{0\} .
$$

If the set $\mathrm{G} \subset[0,4 \pi]$ with Lebesgue measure zero is the set of all zeros of the function $\mathrm{g}$ with infinite multiplicity in $\mathrm{P}$, and if

$$
\int_{0}^{\omega} \ln \mathrm{T}(\mathrm{s}) \mathrm{d} \mu\left(\mathrm{G}_{\mathrm{s}}\right)=-\infty,
$$

where $T(s)=\inf _{k} \frac{\eta_{k} s^{k}}{k !}$ and $\mu\left(G_{s}\right)$ is the Lebesgue measure of s-neighborhood of $G$ and $\omega \in(0,4 \pi)$ is an arbitrary constant, then $\mathrm{g} \equiv 0$ in $\overline{\mathbb{C}}_{+}$.

It follows from (2.4) and (3.4) that

$$
\left|F^{(k)}(z)\right| \leqslant \eta_{k}, \quad k \in \mathbb{N} \cup\{0\},
$$

where

$$
\eta_{k}=B \sum_{r \in q^{N_{0}}}\left(\frac{\ln r}{\ln q}\right)^{k} \exp \left(-\frac{\varepsilon}{2}\left(\frac{\ln r}{\ln q}\right)^{\delta}\right), \quad k \in \mathbb{N}_{0}
$$

and $\mathrm{B}>0$ is a constant.

We can get the estimate for $\eta_{k}$

$$
\eta_{k} \leqslant B \int_{0}^{\infty} x^{k} \exp \left(-\frac{\varepsilon}{2} x^{\delta}\right) d x \leqslant D d^{k} k ! k^{k \frac{1-\delta}{\delta}}
$$

where $\mathrm{D}$ and $\mathrm{d}$ are constants depending on $\mathrm{B}, \varepsilon$ and $\delta$. 
Theorem 3.7. If (3.12) holds, then $\mathrm{Q}_{4}=\varnothing$.

Proof. Using Theorem 3.6, we obtain that the function F satisfies the condition

$$
\int_{0}^{\omega} \ln \mathrm{T}(\mathrm{s}) \mathrm{d} \mu\left(\mathrm{Q}_{4, \mathrm{~s}}\right)>-\infty,
$$

where $T(s)=\inf _{k} \frac{\eta_{k} s^{k}}{k !}, k \in \mathbb{N}_{0}, \mu\left(Q_{4, s}\right)$ is the Lebesgue measure of the s-neighborhood of $Q_{4}$, and $\eta_{k}$ is defined by (3.13). Now we get

$$
\mathrm{T}(\mathrm{s}) \leqslant \mathrm{D} \exp \left\{-\frac{1-\delta}{\delta} \mathrm{e}^{-1} \mathrm{~d}^{-\frac{\delta}{1-\delta}} \mathrm{s}^{-\frac{\delta}{1-\delta}}\right\}
$$

by (3.13). It follows from (3.14) and (3.15) that

$$
\int_{0}^{\omega} s^{-\frac{\delta}{1-\delta}} d \mu\left(Q_{4, s}\right)<\infty .
$$

Since $\frac{\delta}{1-\delta} \geqslant 1$, from (3.16), we obtain that, for arbitrary $s, \mu\left(Q_{4, s}\right)=0$ or $Q_{4}=\varnothing$.

Theorem 3.8. Assume (3.12) holds. Then, the BVP (1.2)-(1.3) has a finite number of eigenvalues and spectral singularities with a finite multiplicity.

Proof. In order to prove the theorem, we need to show that the function $\mathrm{F}$ has a finite number of zeros with finite multiplicities in the region $P$.

Using Theorem 3.3 and Theorem 3.7, we conclude that the set $\mathrm{Q}_{3}=\varnothing$. Thus, the bounded sets $\mathrm{Q}_{1}$ and $\mathrm{Q}_{2}$ do not have accumulation points, that is to say, the function $\mathrm{F}$ has only a finite number of zeros in $\mathrm{P}$. Since $\mathrm{Q}_{4}=\varnothing$, these zeros are of finite multiplicity.

\section{References}

[1] M. Adıvar, E. Bairamov, Spectral properties of non-selfadjoint difference operators, J. Math. Anal. Appl., 261 (2001), 461-478. 1, 1

[2] M. Adıvar, M. Bohner, Spectral analysis of q-difference equations with spectral singularities, Math. Comput. Modelling, 43 (2006), 695-703. 1, 1

[3] M. Adıvar, M. Bohner, Spectrum and principal vectors of second order q-difference equations, Indian J. Math., 48 (2006), 17-33. 1

[4] R. P. Agarwal, Difference equations and inequalities, Theory, methods, and applications, Second edition, Monographs and Textbooks in Pure and Applied Mathematics, Marcel Dekker, Inc., New York, (2000). 1

[5] Y. Aygar, M. J. Bohner, On the spectrum of eigenparameter-dependent quantum difference equations, Appl. Math. Inf. Sci., 9 (2015), 1725-1729. 1

[6] E. Bairamov, A. O. Çelebi, Spectrum and spectral expansion for the non-selfadjoint discrete Dirac operators, Quart. J. Math. Oxford Ser., 50 (1999), 371-384. 1, 3.6

[7] C. Berg, A. Ruffing, Generalized q-Hermite polynomials, Comm. Math. Phys., 223 (2001), 29-46. 1

[8] M. Bohner, A. Peterson, Dynamic equations on time scales, An introduction with applications, Birkhäuser Boston, Inc., Boston, MA, (2001). 1

[9] E. P. Dolzhenko, Boundary value uniqueness theorems for analytic functions, Math. Notes, 25 (1979), 437-442. 3

[10] V. Kac, P. Cheung, Quantum calculus, Universitext, Springer-Verlag, New York, (2002). 1

[11] W. G. Kelley, A. C. Peterson, Difference equations, An introduction with applications, Second edition, Harcourt/Academic Press, San Diego, CA, (2001). 1

[12] A. M. Krall, E. Bairamov, Ö. Çakar, Spectrum and spectral singularities of a quadratic pencil of a Schrödinger operator with a general boundary condition, J. Differential Equations, 151 (1999), 252-267. 1

[13] A. M. Krall, E. Bairamov, O. Cakar, Spectral analysis of non-selfadjoint discrete Schrödinger operators with spectral singularities, Math. Nachr., 231 (2001), 89-104. 1 
[14] B. M. Levitan, I. S. Sargsjan, Sturm-Liouville and Dirac operators, Translated from the Russian, Mathematics and its Applications (Soviet Series), Kluwer Academic Publishers Group, Dordrecht, (1991). 1, 1

[15] V. E. Lyance, A differential operator with spectral singularities, I, II, Amer. Math. Soc. Transl., Ser., 60 (1967), $227-283$. 1

[16] M. A. Naĭmark, Investigation of the spectrum and the expansion in eigenfunctions of a non-selfadjoint differential operator of the second order on a semi-axis, Amer. Math. Soc. Transl., 16 (1960), 103-193. 1 